\title{
Synthesis of oil palm frond (OPF) based silica nanomaterial via sol-gel method with enhanced phenol removal
}

\author{
N. S. Osman, M. L. Ismail, N. Sapawe* \\ Universiti Kuala Lumpur Branch Campus Malaysian Institute of Chemical and Bioengineering Technology (UniKL MICET), Lot 1988 Vendor City, \\ Taboh Naning, 78000 Alor Gajah, Melaka, Malaysia.
}

\begin{abstract}
This research investigates the potential of the oil palm frond (OPF), a well-known biomass from the oil palm industry, as a feasible silica precursor that can be utilised in the removal of phenol from an aqueous solution. Dried OPF was combusted to obtain OPF ash that was treated with citric acid before being synthesised as silica nanomaterial via the sol-gel method. The FTIR results of synthesised silica exhibit a similar peak with commercially available silica. Silica material was then used for phenol removal under different parameters including $\mathrm{pH}$, contact time, dosage, concentration, and temperature, then analysed using UV-Vis Spectrophotometer. The optimum condition was obtained at $\mathrm{pH} 7$ within 45 mins of contact time using $0.2 \mathrm{~g} / \mathrm{L}$ silica dosage under 10 ppm of phenol concentration at $303 \mathrm{~K}$ that aid in enhancing phenol removal by the OPF-based silica. At this condition, silica nanomaterial successfully removed up to $68 \%$ of phenol in an aqueous solution with adsorption capacity of the adsorbent is within the range of $34 \mathrm{mg} / \mathrm{g}$. These results demonstrate the potential application of silica nanomaterial from OPF as an adsorbent in phenol removal from wastewater.
\end{abstract}

\author{
ARTICLE HISTORY \\ Revised: 27 Oct. 2021 \\ Accepted: 1 Nov. 2021 \\ KEYWORDS \\ OPF-based silica \\ Phenol \\ Sol-gel method \\ Wastewater \\ Nanomaterial
}

Received: 12 Sep. 2021

\section{INTRODUCTION}

Several approaches have been studied extensively for the removal of toxic substances from water and can be divided into biological, chemical, and physical treatment. Since the rapid expansion of industrialization, water pollution is arguably the most concerning issue that has affected human life. Water pollutants mainly come from human and industrial waste and consist of heavy metal material, biological contaminants, pharmaceutical contaminants that are dangerous to the ecosystem and living things due to their indestructible nature and acute behaviour [1]. Phenol (benzenol) or also known as hydroxybenzene with molecular formula $\mathrm{C}_{6} \mathrm{H}_{5} \mathrm{OH}$, is an aromatic hydrocarbon group bound to the hydroxyl group (-OH). It has various industrial uses and has been utilized widely in dyes production, pesticides, and drugs as intermediate chemicals [2,3]. In ambient temperature, phenol physical attributes are identified as white/colourless crystalline powder that is hydroscopic in water and slightly in an organic solvent such as alcohol, glycerol and petroleum that make it easier to leak into groundwater sources or water surface [3].

In Malaysia, massive industrialization has caused population growth and rapid urbanization. It has led to the increase of hazardous substance discharge and effluent from a wastewater treatment plant. As a result, a higher level of pollutants including phenol and phenolic compounds were traced as they are easily dissolved in water at room temperature [3-5]. According to National Water Quality Standards for Malaysia, phenol concentration shall not exceed $10 \mathrm{ug} / \mathrm{L}$ in water. Higher phenol concentrations (100-1000 ug/L) produce unpleasant odor and taste that can react with chlorine to form chlorophenol, a carcinogenic and toxic to humans [6].

The introduction of nanomaterials caused literal explosive growth in the evolution of water treatment technology. Its smaller size and larger surface area are beneficial to many interfaces related applications. The chemical, physical, electro and mechanical characteristics can be tuned to control their surface morphology, crystal orientation, shape, size and others [7]. Silica was studied extensively over the years in the development of nanomaterials owing to the marked improvement of their preparation through a sol-gel method and the stable free energy that almost resemblance to crystalline quartz. Furthermore, the $\mathrm{Si}-\mathrm{O}$ bond is strong that the reaction that takes place during the sol-gel process is highly irreversible. The synthesis of amorphous silica also has high thermal stability and the interactions between surfactants can be tuned due to silica ability to be in anionic, cationic, or neutral form depending on the $\mathrm{pH}[8,9]$.

Phenol removal using silica as an adsorbent has been investigated by Roostaei and Tezel [10]. In this study, HiSiv 1000 , an adsorbent that contains crystalline, inorganic silica-alumina structure, displays the best result whereby in the powder form, it has the highest adsorption capacity [10]. The application of commercial porous silica gel was used in a study that investigates the efficiency of polyaniline coated silica gel in removing phenol [11]. Silica aerogel synthesised from the commercial sodium silicate also has been investigated. The results showed that the synthesis silica gel via solgel method could remove phenol in an aqueous solution at $\mathrm{pH} 8.5$ with efficiency up to $90 \%$ when phenol concentration was below $50 \mathrm{ppm}$, and the maximum adsorption capacity was $75.25 \mathrm{mg} / \mathrm{g}$ [12]. Other work has studied the performance 
of amino-modified silica nanoparticles synthesised from TEOS (tetraethyl orthosilicate) via the sol-gel method [11]. In this study, the synthesised silica is categorized based on TEOS concentration used during synthesis and the best adsorbent is AMS-10 with removal capacity calculated to be $35.2 \mathrm{mg} / \mathrm{g}$ [13].

Prior to this, phenol removal using agricultural biomass adsorbent has been investigated which includes the biomass such as coconut, rice husk ash, and sugarcane bagasse which all manifested in ash form and render as adsorbent after activation with various forms of treatment and success in removing phenol [14]. Silica nanomaterial was synthesised from commercially available silica precursors such as TMOS and TEOS, however, a recent trend has seen the emergence of agricultural biomass as silica sources such as rice husk, bamboo culm and corn cob [15-18].

Roslan et al. (2016) suggested an OPF containing siliceous material based on the analysis of its ash contain [19]. Approximately 10 tonnes dry weight of OPF were produced per hectare during the pruning session and this can increase up to 14 tonnes dry weight per hectare during replanting [20]. The abundance of this biomass made it a viable alternative as a silica precursor for the synthesis of silica nanomaterial from agricultural waste.

For this research, an oil palm frond (OPF), biomass from the oil palm industry (plantation) has been utilised as silica precursor in the synthesis of silica nanomaterial via a modified sol-gel method which was later applied as adsorbent material for phenol removal from aqueous solution. Based on previous silica extraction from agricultural waste, silica is usually extracted and produced into powder form before being used as a precursor in silica nanoparticle synthesis. This project aims to utilise sodium silicate solution produced from OPF ash that is treated with nitric acid as silica precursor and directly used in sol-gel synthesis to produce the silica nanoparticle. The method of producing sodium silicate from treated OPF ash has been established based on a study performed previously by this research group [21]. The modification aspect of this study is by directly applying treated OPF ash-based sodium silicate solution into the sol-gel process instead of extracting it as powder silica which is usually performed in a previous study setting.

\section{MATERIALS AND METHODS}

\section{Chemicals}

Oil palm frond (OPF) was collected from a private palm oil plantation at Alor Gajah, Melaka. The OPF ash was treated using citric acid from HmBG. Reagents used in sol-gel including $\mathrm{NaOH}$ pellet from VChem, hydrochloric acid from Sigma-Aldrich (37\%), and hexadecyltrimethylammonium bromide (CTAB) from Sigma Aldrich (98\% purity) while phenol powder was purchased from Merck Millipore (99\% purity). $\mathrm{SiO}_{2}$ reagent grade purchase from Merck Millipore ( $98 \%$ purity).

\section{Preparation of raw material (OPF) and acid leaching treatment}

The collected OPF was cleaned, sorted, and cut before being dried in the oven at $313 \mathrm{~K}$ for 24 hours. The OPF was then combusted in an electric muffle furnace at $873 \mathrm{~K}$ for 3 hours to obtain oil palm frond ash (OPFA). Citric acid with a concentration of $3 \%$ was first prepared. $20 \mathrm{~g}$ of OPFA was measured on an analytical balance and poured into the citric acid solution. The mixture was placed in the hot place at $343 \mathrm{~K}$ with a reaction time of 1 hour. The treated ash was rinsed using distilled water at room temperature to remove citric acid from the ash then followed with drying at $353 \mathrm{~K}$ for $8 \mathrm{~h}$ in the drying oven. Dried treated ash was finally calcined at $1073 \mathrm{~K}$ for 30 minutes in the electric muffle furnace [21].

\section{Silica nanomaterial synthesis}

Silica was prepared at ambient temperature by mixing acid-leached OPFA in $2 \mathrm{M} \mathrm{NaOH}$ for 1 hour with continuous stirring. The direct application of treated OPF-based sodium silicate solution as silica precursor is the modification in this sol-gel process. Previous study agricultural waste silica was extracted into powder form before being applied in silica synthesis using the sol-gel method. CTAB was dissolved in distilled water and stirred on a hot plate at $500 \mathrm{rpm}$ for 30 mins at room temperature. Sodium silicate solution was then added gradually and where it turned into a murky whitish solution. $\mathrm{HCl}$ acid was used to obtain $\mathrm{pH}$ between 10.37 and 10.88. This range is the best $\mathrm{pH}$ for the promotion of silica nanoparticles during the gelling period. The solution was continuously stirred at $800 \mathrm{rpm}$ for another 1 hour at $353 \mathrm{~K}$. The solution was aging at $333 \mathrm{~K}$ overnight. The solution was filtered, and the silica nanomaterial was repeatedly rinsed with water-methanol solution (1:1) and dried at $373 \mathrm{~K}$. The white powder silica was then calcined at $773 \mathrm{~K}$ for 5 hours at $278 \mathrm{~K} / \mathrm{min}$ rate. Silica nanomaterial obtained was characterized using FTIR (Thermo Scientific Nicolet iS10).

The following equations were used to calculate the corresponding parameters.

$$
\frac{O P F_{a}}{O P F_{i}} \times 100=\% \text { of } O P F \text { ash obtained }
$$

where $\mathrm{OPF}_{\mathrm{i}}$ represent the initial amount of dry $\mathrm{OPF}$ before combustion and $\mathrm{OPF}_{\mathrm{a}}$ represent the OPF ash obtain after combustion,

$$
\frac{O P F_{t}}{O P F_{a}} \times 100=\% \text { of acid leashed } O P F \text { ash obtained }
$$

where $\mathrm{OPF}_{\mathrm{a}}$ represent the initial amount of OPF ash before acid leaching and $\mathrm{OPF}_{\mathrm{t}}$ represent the amount of OPF ash after acid leaching,

$$
\frac{O P F-\text { based silica }}{O P F_{t}} \times 100=\% \text { of silica nanoparticle obtained }
$$


where $\mathrm{OPF}_{\mathrm{t}}$ represents the amount of OPF ash after acid leaching and OPF-based silica is the amount of silica nanoparticle obtained from the synthesis.

\section{Phenol removal from aqueous solution}

Silica nanomaterial obtained was used as an adsorbent in phenol removal. Performance analyses were performed at different $\mathrm{pH}(3,5,7,9,11)$, contact time $(5,10,15,30,45,60 \mathrm{~min})$, silica dosage $(0.2,0.4,0.5,0.8,1.0 \mathrm{~g} / \mathrm{L})$, phenol concentration $(10,20,30,50,70,100 \mathrm{ppm})$, and temperature $(303 \mathrm{~K}, 323 \mathrm{~K}, 343 \mathrm{~K})$; with continuous stirring on hot plate (400 rpm). The results were analysed using UV-Vis (Perkin-Elmer Lambda $35 \mathrm{UV}-\mathrm{Vis}$ ) at $\lambda$ max $267 \mathrm{~nm}$. A standard calibration curve was initially produced to obtain a linear equation to calculate the concentration of phenol in an aqueous solution after removal.

The percentage removal of phenol using silica nanomaterial absorbent is measured and its efficiency is calculated as follows:

$$
\frac{C_{i}-C_{f}}{C_{i}} \times 100=\% \text { of phenol removal }
$$

where $C_{\mathrm{i}}$ is the initial concentration and $C_{\mathrm{f}}$ is the final concentration. The adsorption capacity were then calculated such that

$$
\frac{\left(C_{i}-C_{f}\right) V}{W}=\text { Adsorption capacity }(q)
$$

where $V$ is the volume of the phenol used during adsorption process $\left(\mathrm{cm}^{3}\right), W$ is the mass of adsorbent used during the adsorption process $(\mathrm{mg})$ and $q$ is the amount adsorbed in unit mas of the adsorbent $(\mathrm{mg} / \mathrm{g})$.

\section{EXPERIMENTAL RESULTS}

\section{Silica nanomaterial synthesis from OPF and characterization}

In the synthesis of silica nanomaterial from OPF as silica precursor, modified sol-gel methods were applied and CTAB was chosen as the surfactant that renders the sodium silicate obtained from treated OPF ash to be a white colour powder. Figure 1 provides the overall process of synthesising the silica nanomaterials. Table 1 shows the yield percentage of silica nanomaterial obtained per 1000 gm of dry weight OPF.

Table 1. Percentage yield of silica nanomaterial from OPF.

\begin{tabular}{ccccccr}
\hline Dry OPF (g) & OPF Ash (g) & OPF Ash (\%) & $\begin{array}{c}\text { Acid Leached } \\
\text { OPF Ash (g) }\end{array}$ & $\begin{array}{c}\text { Acid Leached } \\
\text { OPF Ash (\%) }\end{array}$ & Silica (g) & Silica (\%) \\
\hline 1,000 & 45 & 4.5 & 21.6 & $48 \%$ & 14.04 & 65 \\
\hline
\end{tabular}

The results show approximately $65 \%$ silica nanomaterial yield and are relatively comparable to the yield of silica from other agricultural biomass such as sugarcane bagasse ash (50\%-80\%), bamboo leaf (60\%-80\%), wheat straw (50\%-55\%) and OPA $(40 \%-80 \%)[15,16]$. It is also slightly higher when compared to the yield of silica from sources such as corn cob, bamboo culm and cassava peel [15].

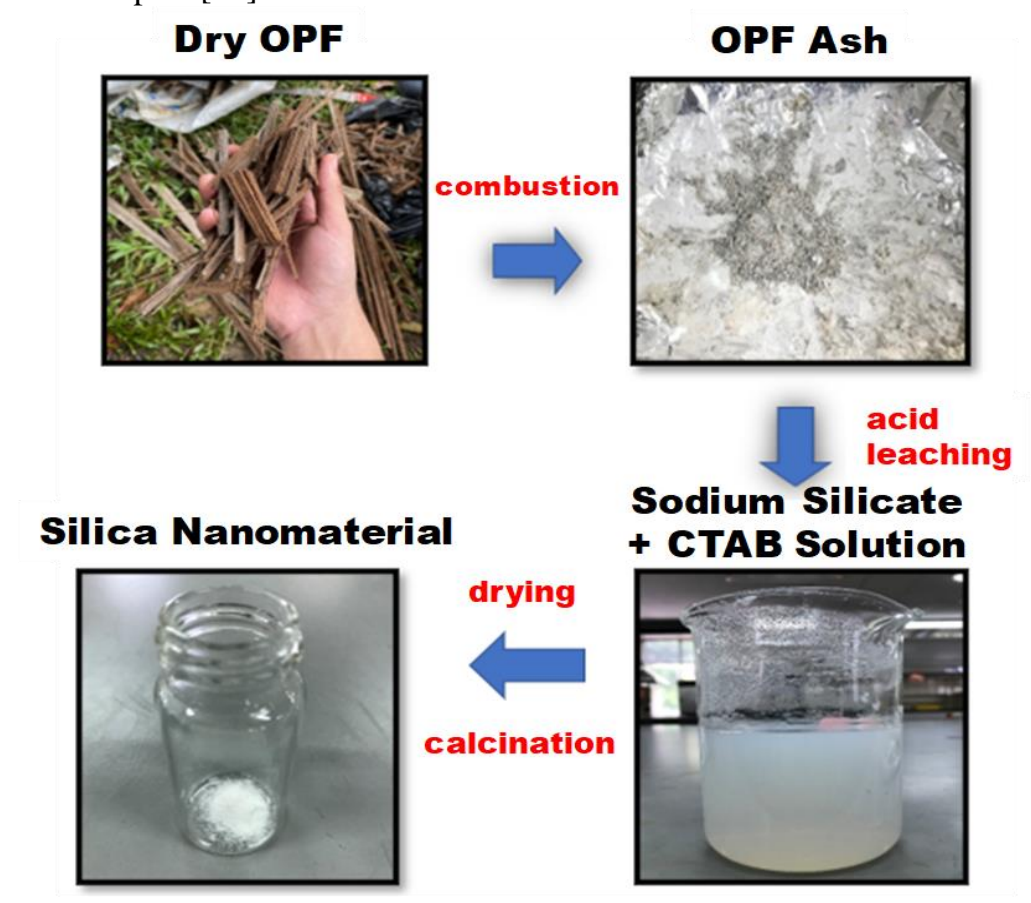

Figure 1. Silica nanomaterial synthesis from OPF. 
Silica nanomaterial from OPF was analysed using FTIR and the result was shown in Figure 2. The spectra of the silica obtained from OPF display similar peaks to commercial silica when examined within the range of $4000-400 \mathrm{~cm}^{-1}$ For both silicas, the presence of a strong and broad band around $3420-3440 \mathrm{~cm}^{-1}$ is due to the $-\mathrm{OH}$ stretching vibration of the silanol or the adsorbed water molecules on the silica surface. The next peak within the range of $1620-1640 \mathrm{~cm}^{-1}$ represents the bending vibration band of the trapped $\mathrm{H} 2 \mathrm{O}$ molecule within the silica matrix. The strong band around $1060-1110 \mathrm{~cm}^{-}$ ${ }^{1}$ corresponds to the asymmetric vibration of the $\mathrm{Si}-\mathrm{O}-\mathrm{Si}$ siloxane bond, the bond that forms the silica matrix backbone.

The peak at $941 \mathrm{~cm}^{-1}$ and $451 \mathrm{~cm}^{-1}$ represents the valent and deformation vibrations of the Si-O-Si siloxane bond that were absent in OPF-based silica however this bond is present by the strong peak at 640 [18]. The absence of both peaks in OPF-based silica might be due to the water content in OPF-based silica that is smaller compared to the commercial silica [18]. FTIR spectra obtain from the OPF-based indicate the presence of the essential functional group that was also present in FTIR spectra for the synthesise of silica nanomaterials from agricultural waste [16,17].

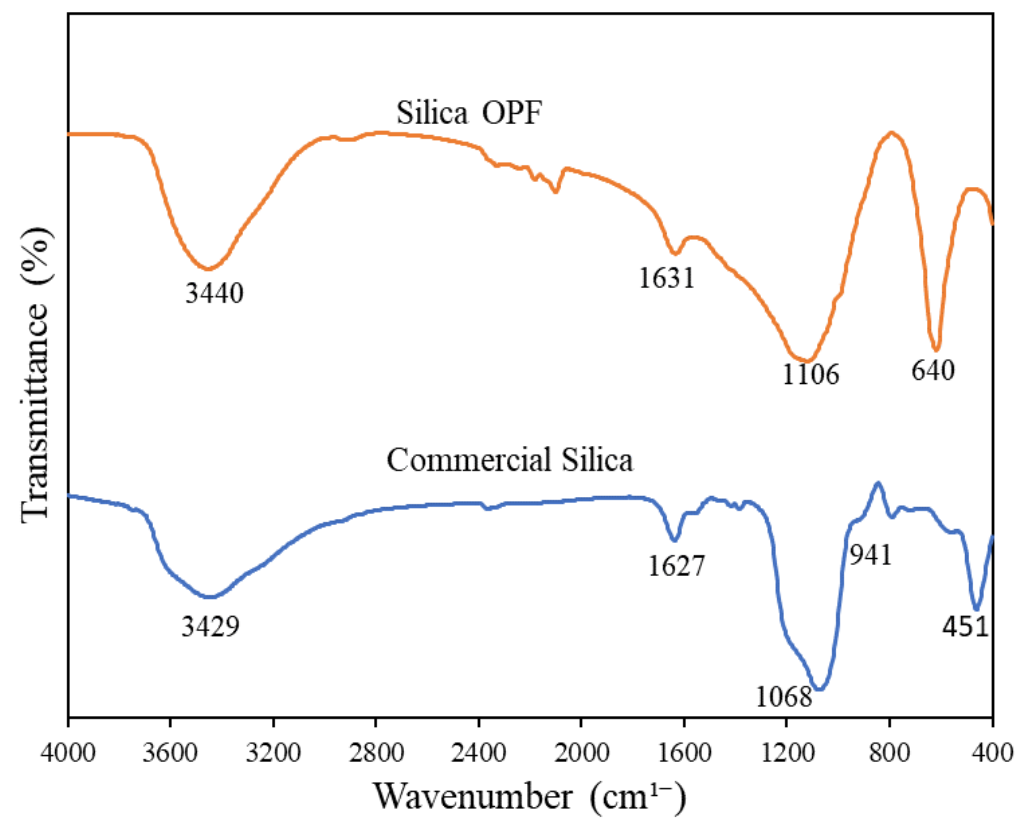

Figure 2. FTIR spectra of silica nanomaterial.

\section{Effect of different parameters in phenol removal using OPF-based silica}

The removal of phenol using silica nanomaterial from OPF was performed and analysed using UV-Vis by measuring maximum absorption at $267 \mathrm{~nm}$. The efficiency was investigated through various parameters including $\mathrm{pH}$, contact time, silica dosage, phenol concentration, and temperature as shown in Figure 3.

The $\mathrm{pH}_{\mathrm{zpc}}$ of silica were analysed using $\mathrm{pH}$ titrations at different strengths that provide the value of 6.45. The result shows that the best $\mathrm{pH}$ is 7 where the equilibrium was reached within 30-45 mins. There is no significant reduction after 45 mins contact time. At this condition, phenol was removed up to $63 \%$ with adsorption capacity $(q)$ at $31.3 \mathrm{mg} / \mathrm{g}$. However, at acidic and basic conditions, phenol removal was significantly lower with only 25-26\% with adsorption capacity $(q)$ of $11-13 \mathrm{mg} / \mathrm{g}$. These findings supported the results obtained by Rahmani et al. [12] and Saleh et al. [13] where the highest removal of phenol was recorded at $\mathrm{pH} 8.5$ and $\mathrm{pH} 6-7$, respectively.

Phenol has a pKa value of approximately 10, indicating an anionic form while OPF-based silica has a $\mathrm{pH}_{\mathrm{zpc}} \mathrm{Value}$ of 6.45 indicating negative sides on its surface. This could be the reason for the low removal of phenol at a higher $\mathrm{pH}$ value. Thus, at higher $\mathrm{pH}$, electrostatic repulsive force occurs between the negative surface of OPF-based silica and the phenoxide ion and prevents the adsorption of phenol onto the silica nanoparticle. Meanwhile, for phenol solution on $\mathrm{pH}$ lower than 11, phenol maintains its dissociative form and is relatively attracted to the negative side of OPF based silica $(6.45<\mathrm{pH}<11)$ and positive surface $(3<\mathrm{pH}<6.45)$ due to dispersion interaction. Lower phenol removal at low $\mathrm{pH}$ might be due to the competition occurring on the OPF-based silica surface between proton ions and phenol molecules [12]. This also could explain the adsorption capacity of OPF-based silica which increases as $\mathrm{pH}$ increases but decreases when $\mathrm{pH}$ is more than 10 .

The effect of silica dosage was investigated between $0.2 \mathrm{~g} / \mathrm{L}$ and $1.0 \mathrm{~g}$ and the results are shown in Figure 3(c). It demonstrates a higher phenol removal when the dosage is increased. Nonetheless, the increment of phenol removal at a higher dosage does not give a significant difference which might be due to the saturation of silica at a higher dosage and the phenol removal reaching equilibrium at a faster rate. This result indicates that this silica material working mechanism in removing phenol may not be driven based on its dosage in the aqueous solution.

The effect of phenol concentration was investigated from between 10 and $100 \mathrm{ppm}$. From the result, phenol removal shows a significant decrease as the phenol concentration increases. It is highly improbable that the phenol adsorption by silica is driven based on phenol concentration, as though the removal percentage decrease; however, the adsorption 
capacity $(q)$ by the silica is retained almost at a similar rate even at a higher phenol concentration. The effect of temperature shows that phenol removal is significantly higher at room temperature $(303 \mathrm{~K})$ with the highest adsorption capacity $(q)$ of silica is within the range of $33-36 \mathrm{mg} / \mathrm{g}$. Silica nanomaterial adsorption capacity $(q)$ calculated from this study is comparable to other studies performed utilising amino-modified silica [15].
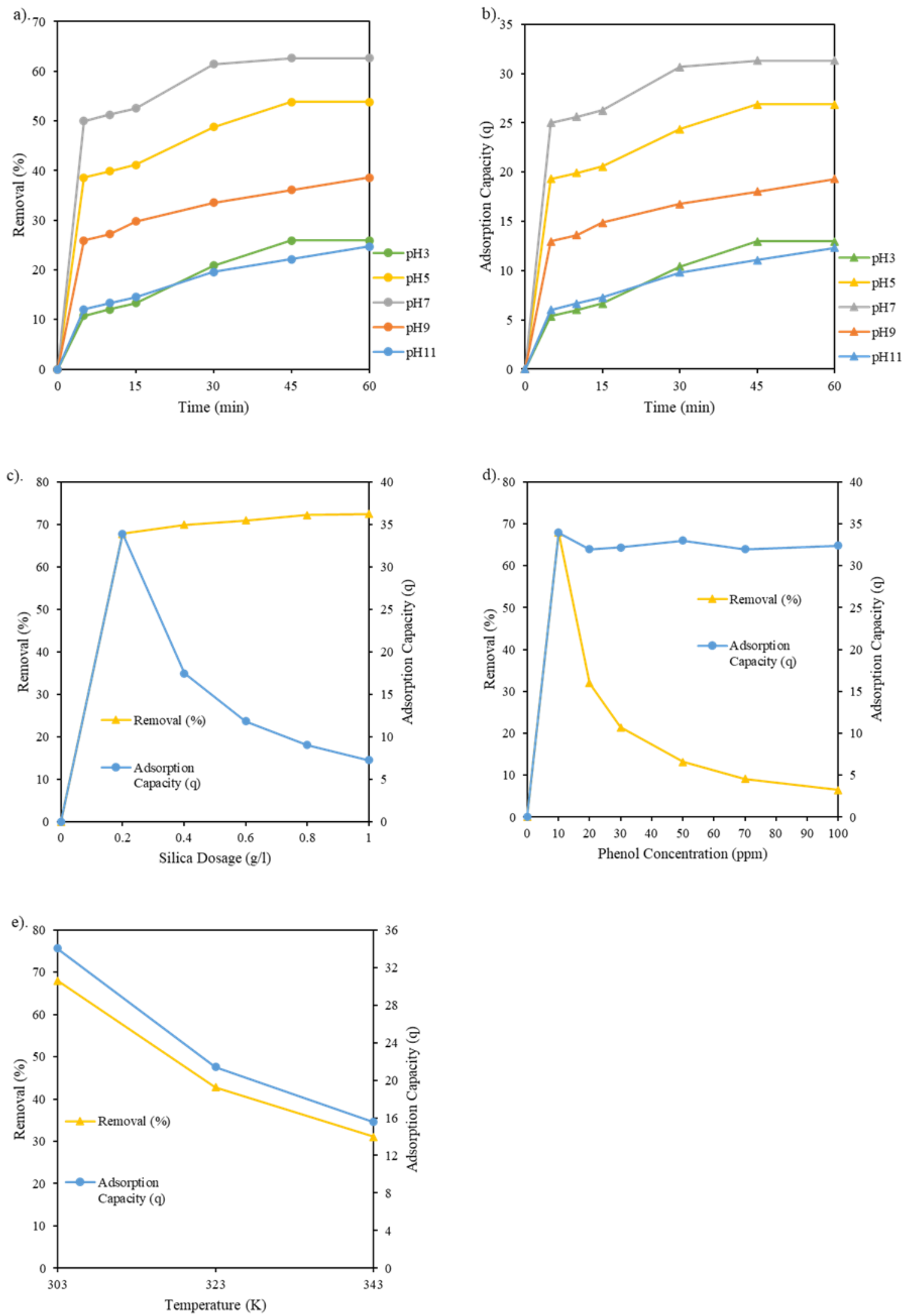

Figure 3. Removal of phenol as a function of several parameters: (a,b) pH and contact time (c) silica dosage (d) phenol concentration (e) temperature. 


\section{Silica nanomaterial performance in phenol removal}

The efficiency of phenol removal by the OPF-based silica nanomaterial was performed in batches at optimum $\mathrm{pH} 7$, contact time $(45 \mathrm{~min})$, silica dosage $(0.2 \mathrm{gm} / \mathrm{l})$, phenol concentration $(10 \mathrm{ppm})$, and temperature $(303 \mathrm{~K})$. The data tabulated in Table 2 shows the removal efficiency at $68 \%$ and the adsorption capacity $(q)$ is significantly constant at 34 $\mathrm{mg} / \mathrm{g}$ at the optimum parameters. These results indicate that the silica synthesise from OPF has a feasible application in phenol removal from an aqueous solution as it efficiently lowered phenol concentration. The maximum adsorption capacity obtained by this study is almost comparable to Saleh et al. that utilised amino-modified silica synthesised from TEOS [13]. Nonetheless, the result from this work is slightly lower when compared to the results obtained by Rahmani et al. [12].

Table 2. Adsorption of phenol by OPF-based silica nanomaterial at optimum condition [pH 7, 45 mins of contact time, $0.2 \mathrm{~g} / \mathrm{L}$ silica dosage, $10 \mathrm{ppm}$ concentration of phenol, and $303 \mathrm{~K}$ ]

\begin{tabular}{ccccc}
\hline Batch & Before $(\mathrm{ppm})$ & After $(\mathrm{ppm})$ & Removal $(\%)$ & Adsorption Capacity $(\mathrm{mg} / \mathrm{g})$ \\
\hline 1 & 10 & 3.183 & 68.170 & 34.085 \\
\hline 2 & 10 & 3.283 & 67.170 & 33.585 \\
\hline 3 & 10 & 3.198 & 68.020 & 34.010 \\
\hline
\end{tabular}

The adsorption capacity $(q)$ ability by OPF-based silica display a higher feasible adsorption interaction as its viable mechanism in removing phenol from the investigated solution. The synthesised silica tested in different batches exhibit similar results with the literature when the optimum parameter was utilised. This provides a probable indication that the parameter investigation has an effect in optimizing the working mechanism that allows OPF-based silica to remove phenol from an aqueous solution.

\section{CONCLUSION}

The utilisation of OPF as a silica precursor in this research project has shown a positive result when applied as feasible adsorbent material in removing phenol from the aqueous solution. The FTIR spectra from the OPF-based silica display significant similarity with the FTIR spectra of commercially available reagent grade silica. Sodium silicate solution obtained from treated OPF ash was successfully synthesised into silica nanomaterial where CTAB was applied as surfactant via modified sol-gel method. Subsequently, synthesised material was analysed for its feasibility as adsorbent material in removing phenol, a widely used intermediate chemical in various industries. OPF-based silica was analysed at different parameters including $\mathrm{pH}$, contact time, dosage, phenol concentration, and temperature. At optimum

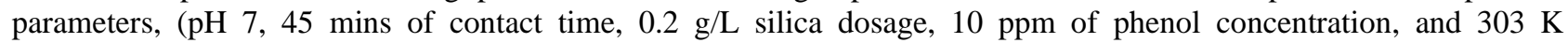
temperature), synthesised silica significantly removed up to $68 \%$ phenol from aqueous solution, and retain constant adsorption capacity at $34 \mathrm{mg} / \mathrm{g}$. Therefore, this research resulted in an open plethora viability of the utilisation of OPF as silica precursor in nanomaterial synthesis as well as possible application in toxic pollutant removal in wastewater.

\section{ACKNOWLEDGEMENT}

The authors are grateful for the financial support by the Fundamental Research Grant Scheme (FRGS/1/2019/STG07/UNIKL/02/1) from Ministry of Education Malaysia (MOE) and the Universiti Kuala Lumpur Branch Campus Malaysian Institute of Chemical and Bioengineering Technology (UniKL MICET) for their support.

\section{REFERENCES}

[1] T. Distefano and S. Kelly, “Are we in deep water? Water scarcity and its limits to economic growth", Ecol. Econ., vol. 142, pp. 130-147, 2017, doi: 10.1016/j.ecolecon.2017.06.019.

[2] S. Wasi, S. Tabrez and M. Ahmad, "Toxicological effects of major environmental pollutants: An overview", Environ. Monit Assess., vol. 185, no. 3, pp. 2585-2593, 2013, doi: 10.1007/s10661-012-2732-8.

[3] A. A. Gami, M. Y. Shukor, K. A. Khalil, F. A. Dahalan, A. Khalid and S. A. Ahmad, "Phenol and its toxicity", J Env. Microbiol. Toxico., vol. 2. no. 1, pp. 11-24, 2014.

[4] C. H. Chan and P. E. Lim, "Evaluation of sequencing batch reactor performance with aerated and unaerated fill periods in treating phenol-containing wastewater", Bioresour. Technol., vol. 98, no. 7, pp. 1333-1338, 2007, doi: 10.1016/j.biortech.2006.05.033.

[5] Y. J. Liu, M. Nikolausz and X. C. Wang, "Biodegradation and detoxication of phenol by using free and immobilized cells of Acinetobacter sp. XA05 and Sphingomonas sp. FG03”, J. Environ. Sci. Health A., vol. 44, no. 2, pp. 130-136, 2009, doi: $10.1080 / 10934520802539673$.

[6] J. Sun, X. Liu, F. Zhang, J. Zhou, J. Wu, A. Alsaedi, T. Hayat and J. Li, "Insight into the mechanism of adsorption of phenol and resorcinol on activated carbons with different oxidation degrees", Colloids Surf. A Physicochem. Eng. Asp., vol. 563, pp. 22-30, 2019, doi: 10.1016/j.colsurfa.2018.11.042Z.

[7] Z. Li, D. V. Potapenko and R. M. Osgood, "Controlling surface reactions with nanopatterned surface elastic strains", ACS Nano, vol 9, no. 1, pp. 82-87, 2015, doi: 10.1021/nn506150m.

[8] N. Lieu Le and S. P. Nunes, "Materials and membrane technologies for water and energy sustainability", Sustain. Mater. Technol., vol. 7, pp. 1-28, 2016, doi: 10.1016/j.susmat.2016.02.001. 
[9] M. L. Mastronardi, K. K. Chen, K. Liao, G. Casillas and G. A. Ozin, "Size-dependent chemical reactivity of silicon nanocrystals with water and oxygen”, J. Phys. Chem. C, 2015, vol. 119, no. 1 pp. 826-834, 2015, doi: 10.1021/jp510592j.

[10] N. Roostaei and F. H. Tezel, "Removal of phenol from aqueous solution by adsorption", J. Environ. Manage., vol. 70, pp. 15164, 2004, doi: 10.1016/j.jenvman.2003.11.004.

[11] F. belaib, A.H Meniai and M.B Lehocine, "Elimination of phenol by adsorption onto mineral/polyaniline composite solid support", Energy Procedia, vol. 18, pp. 1254-1260, 2012, doi: 10.1016/j.egypro.2012.05.141.

[12] A. Rahmani, F. Nazemi, F. Barjasteh-Askari and M. Davoudi, "Preparation, characterization, and application of silica aerogel for adsorption of phenol: An in-depth isotherm study", Health Scope, vol. 7, no. 3, pp. 1-12, 2018, doi: 10.5812/jhealthscope.15115.

[13] S. Saleh, A. Younis, R. Ali and E. Elkady, "Phenol removal from aqueous solution using amino modified silica nanoparticles" Korean J. Chem. Eng., vol. 36, pp. 529-539, 2019, doi: 10.1007/s11814-018-0217-3.

[14] G. Issabayeva, Y.H, Shu, M.C, Wong, and M.K Aroua, "A review on the adsorption of phenol from wastewater onto diverse groups of adsorbents", Rev. Chem. Eng., vol. 34, no. 6, pp 1-19, 2017, doi: 10.1515/revce-2017-0007.

[15] N. S. Osman and N. Sapawe," Waste material as an alternative source of silica precursor in silica nanoparticle synthesis-A review”, Mater. Today: Proc., vol. 19, pp. 1267-1272, 2019, doi: 10.1016/j.matpr.2019.11.132.

[16] V. L. Le, C. N. Ha Thuc, and H. H. Thuc, "Synthesis of silica nanoparticles from Vietnamese rice husk by sol-gel method", Nanoscale Res. Lett., vol. 8, no. 1, pp. 58-67, 2013, doi: 10.1186/1556-276X-8-58..

[17] S. Norsuraya, H. Fazlena, and R. Norhasyimi, 2016. "Sugarcane bagasse as a renewable source of silica to synthesize Santa Barbara Amorphous-15 (SBA-15)”, Procedia Eng., vol. 148, pp. 839-846, 2016, doi: 10.1016/j.proeng.2016.06.627.

[18] L. A. Zemnukhova, A. E. Panasenko, A. P. Artemyanov and E. A. Tsoy, "Dependence of porosity of amorphous silicon dioxide prepared from rice straw on plant variety", Bioresources, vol. 10, no. 2, pp. 3713-3723, 2015, doi: 10.15376/biores.10.2.3713-3723.

[19] A. M. Roslan, M. A. K. M. Zahari, M. A. Hassan and Y. Shirai, "Investigation of oil palm frond properties for use as biomaterials and biofuels", Trop. Agric. Dev., vol. 56, no. 1, pp. 26-29, 2014, doi: 10.11248/jsta.58.26.

[20] S. K. Loh, "The potential of the Malaysian oil palm biomass as a renewable energy source", Energy Convers. Manag., vol 141, pp. 285-298, 2017, doi: 10.1016/j.enconman.2016.08.081.

[21] N. S. Osman and N. Sapawe, "High purity and amorphous silica $\left(\mathrm{SiO}_{2}\right)$ prepared from oil palm frond (OPF) through sol-gel method”, Mater. Today: Proc., vol.31, no. 1, pp. 228-231, 2020, doi: 10.1016/j.matpr.2020.05.299. 